\title{
TORCICOLO ESPASMÓDICO PÓS-TRAUMÁTICO
}

\author{
MABEL SATOMI KAWASAKI*, OTTO J. HERNÁNDEZ-FUSTES **, \\ EMILIO J. SCHEER NETO***, OTTO HERNÁNDEZ-COSSIO***
}

\begin{abstract}
RESUMO - A distonia pós-traumática (DPT) é sequela rara do traumatismo crânio-encefálico (TCE). Descrevemos um paciente com torcicolo espasmódico e revisamos 31 casos descritos na literatura. O intervalo de tempo entre o TCE e o aparecimento da distonia variou de 2 horas a 9 anos. Em 11 casos o TCE foi de média gravidade e em 20 foi severo. As lesões na tomografia de crânio (CT) foram: hematomas extra e subdurais e hemorragias talâmicas. Na ressonância magnética a lesão mais frequente estava localizada no gânglio basal contralateral ou no tálamo. Em nosso caso, a CT de crânio evidenciava higroma bilateral e edema cerebral difuso, com reabsorção 6 dias depois; a RMN encefálica realizada 1 ano após o TCE mostrou discreta atrofia cerebral. Apesar da fisiopatologia da DPT não estar bem esclarecida, há indícios de disfunção do circuito lentículo-talâmico e que a variação de tempo entre o TCE e o desenvolvimento da DPT, ocorra por presença de neurônios aberrantes.
\end{abstract}

PALAVRAS-CHAVE: distonia pós-traumática, trauma crânio-encefálico, torcicolo espasmódico.

\section{Post traumatic spasmodic torticollis}

ABSTRACT - Dystonia is a rare consequence of head trauma. We describe one case of post-traumatic spasmodic torticollis and review thirty-one cases reported in the literature. The time course among the head injury and the onset of dystonia ranged from two hours to nine years. Eleven cases had mild head injury and twenty had severe. On CT studies, the most frequent lesion was extradural, subdural and thalamic hemorrhagies; on MRI there were lesions in contralateral basal ganglia or thalamus. In our case, initial CT findings were bilateral hygroma and generalized brain edema. A follow-up CT sixt days after head injury showed cerebral atrophy and MRI scan (one year later) showed cerebral atrophy either. The pathophysiologic mechanisms related to the appearence of dystonia are poorly understood. However, there are evidences suggesting that some dysfunction of lenticulothalamic neuronal circuits plays an important role. The period of time between the head injury and the appearence of posttraumatic dystonia, seems to be related to aberrant central neurons.

KEY WORDS: post-traumatic dystonia, head trauma, spasmodic torticollis.

As primeiras menções sobre a importância do trauma no sistema nervoso central (SNC) foram feitas por Galeno (130-200 d.C. ${ }^{1}$ Galeno estudou as consequências do trauma no SNC em gladiadores e, também com base em suas dissecções de animais, concluiu que os distúrbios pós-traumáticos eram de dois tipos: um ligado as sensações e percepções, outro ao controle dos movimentos; sendo estes relacionados a lesões no cérebro e no cerebelo respectivamente. As descrições mais substanciais de pacientes com distonia, apareceram no final do século XIX. Gowers, em 1888, descreveu dois irmãos com movimentos anormais lentos e persistentes, utilizando o termo "coréia tetanóide" 2 . Estes pacientes mais tarde seriam reconhecidos como portadores da doença de Wilson. Nessa mesma época foram levantadas várias hipóteses de que os distúrbios psicológicos seriam fatores importantes

Serviço de Neurologia do Hospital Universitário Cajuru da Pontifícia Universidade Católica (PUC) do Paraná: *Residente de Neurologia; **Chefe do Serviço de Neurologia; ***Neurologista. Aceite: 9-fevereiro1998.

Dr. Otto J. Hernández Fustes - Serviço de Neurologia, Hospital Universitário Cajuru PUC - Av. São José 300 80050-350 Curitiba PR - Brasil. 
Tabela 1. Classificação da distonia.

I. Etiologia
Primárias
Hereditárias
Autossômica dominante
Ligada ao cromossomo X
Esporádicas
Secundárias
Associada a processos neurodegenerativos
Esporádicas
Hereditárias
Associada a distúrbios metabólicos
Aminoácidos
Lípides
Outras
Associada a causas específicas
Lesões perinatais e Kernicterus
Infecções
Síndrome paraneoplásica
Lesões cerebrovasculares
Traumatismos crânio-encefálicos
Toxinas
Drogas
Distonia psicogênica

\section{Idade de início}

Infância - 0-12 anos

Adolescência - 13-20 anos

Adulto - > 20 anos

\section{Distribuição}

Focal

Segmentar

Multifocal

Generalizada

Hemidistonia na gênese de tais movimentos anormais, sendo consagrados outros termos, como por exemplo: "neurose de torção", "espasmos histéricos", "mioclonia do tronco" e "cãimbras tônicas". Em 1908, Schwalbe descreveu minuciosamente uma síndrome observada em três irmãos, com padrão de transmissão autossômica dominante, caracterizada por "cãimbras tônicas e sintomas histéricos", que mais tarde seria conhecida como distonia generalizada hereditária. Esses mesmos movimentos descritos por Schwalbe, foram observados em seis pacientes por Oppenheim em $1911^{3}$, que argumentou ser a causa primária orgânica e não psicogênica ${ }^{3}$. Oppenheim descreveu esses movimentos como espasmos tônicos alternados com hipotonia, com comprometimento da marcha, movimentos de torção do tronco e dos membros, abalos musculares rápidos e nos casos mais graves, deformidades posturas fixas, sendo introduzidos os termos: "dystonia musculorum deformans" enfatizando as alterações do tono muscular e a produção de deformidades posturais; e quando havia proeminência dos distúrbios de marcha, "dysbasia lordotica progressiva". No mesmo ano, Flatau e Sterling criticaram a terminologia usada por Oppenheim, pois as flutuações do tono muscular nem sempre estavam presentes e nem todos os pacientes observados tinham evidências de comprometimento primário muscular, tampouco apresentavam deformidades ${ }^{4}$.

Apesar da conceituação clínica da distonia ser debatida ao longo das últimas décadas, o termo distonia é universalmente aceito. A distonia ${ }^{5,6}$ é

condição neurológica caracterizada por contrações musculares mantidas frequentemente, causando movimentos repetitivos ou de torção, ou posturas anormais. As distonias podem ocorrer durante o repouso ou apenas durante o movimento voluntário, tendem a intensificar-se com a fadiga, o estresse e outros fatores emocionais e a aliviar-se com o sono leve, relaxamento e com o toque delicado na área afetada. A distonia desaparece por completo nas fases profundas do sono. Não há, até o presente momento, marcadores bioquímicos, patológicos ou radiográficos para distonia, sendo o diagnóstico fundamentado pelo reconhecimento do quadro clínico. A classificação da distonia costuma ocorrer com base em três critérios: etiologia, idade de início e distribuição (Tabela 1) (4,7 $^{\text {. }}$.

O objetivo deste estudo é relatar um caso de distonia pós traumática, do tipo torcicolo espasmódico e revisar 31 casos descritos na literatura.

\section{RELATO DE CASO}

MDM, 33 anos, masculino, branco, solteiro, jardineiro, natural de Santa Rosa-RS e procedente de CuritibaPR, com duração do internamento de sete dias (10 a 16-janeiro-1997); apresentou no momento da admissão queixa de movimentos involuntários de torção e hiperextensão da cabeça e do pescoço, com intensificação dos movimentos ao adotar a posição sentada ou ortostática e nos períodos de estresse físico e/ou emocional, aliviandose no repouso em decúbito dorsal e durante o sono. Estes movimentos involuntários anormais iniciaram-se 10 
Tabela 2.Características das distonias pós-traumáticas na literatura revisada.

\begin{tabular}{|c|c|c|c|c|c|}
\hline Autor & & Sexo & $\begin{array}{l}\text { Idade } \\
\text { (anos) }\end{array}$ & $\begin{array}{l}\text { Severidade } \\
\text { do trauma }\end{array}$ & Sítio da Lesão \\
\hline O' Callaghan, 1962 & 1 & $\mathrm{~F}$ & 10 & M & - \\
\hline Messimy et al., $1979^{14}$ & 2 & M & 21 & M & calcificação do núcleo caudado D \\
\hline Burke et al., $1980^{15}$ & 3 & $\mathrm{~F}$ & 2 & $\mathrm{~S}$ & afundamento de crânio $\mathrm{E}$ \\
\hline \multirow[t]{2}{*}{ Maki et al., $1980^{16}$} & 4 & M & 10 & M & infarto de gânglios da base D \\
\hline & 5 & M & 10 & M & infarto de gânglios da base D \\
\hline Mauro et al., $1980^{17}$ & 6 & $\mathrm{~F}$ & 8 & M & - \\
\hline Brett \& Hoare, $1981^{18}$ & 7 & $\mathrm{~F}$ & 5 & M & $\begin{array}{l}\text { faixa estreita de atenuação } \\
\text { estendendo-se do PE ao BPCI }\end{array}$ \\
\hline Demierre \& Rondot, $1983^{19}$ & 8 & $\mathrm{~F}$ & 3 & $\mathrm{M}$ & $\begin{array}{l}\text { lesão hipodensa envolvendo CNCE, } \\
\text { PE e BACI }\end{array}$ \\
\hline Andrew $\mathbf{J}$ et al. ${ }^{20}$ & 9 & M & 8 & $\mathrm{~S}$ & lesão no NC, NL e BACI \\
\hline \multirow[t]{3}{*}{ Pettigrew \& Jankovic, $1985^{21}$} & 10 & M & 7 & $\mathrm{~S}$ & normal \\
\hline & 11 & M & 17 & $\mathrm{~S}$ & encefalomalacia GPD \\
\hline & 12 & $\mathrm{~F}$ & 62 & M & $\begin{array}{l}\text { infarto do tâlamo posterior D, } \\
\text { sela vazia }\end{array}$ \\
\hline Eaton, $1988^{22}$ & 13 & M & 56 & M & hematoma subdural de hemisfério $\mathrm{E}$ \\
\hline Isaac \& Cohin, $1989^{23}$ & 14 & M & 28 & $\mathrm{~S}$ & hematoma de gânglios basais \\
\hline \multirow[t]{7}{*}{ Krauss et al., $1992^{24}$} & 15 & $\mathrm{~F}$ & 7 & $\mathrm{~S}$ & $?$ \\
\hline & 16 & $\mathrm{~F}$ & 9 & M & $?$ \\
\hline & 17 & M & 6 & $\mathrm{~S}$ & $?$ \\
\hline & 18 & M & 3 & $\mathrm{~S}$ & $?$ \\
\hline & 19 & $\mathrm{M}$ & 13 & $\mathrm{~S}$ & $?$ \\
\hline & 20 & $\mathrm{M}$ & 4 & $\mathrm{~S}$ & $?$ \\
\hline & 21 & $\mathrm{M}$ & 16 & $\mathrm{~S}$ & $?$ \\
\hline \multirow[t]{10}{*}{ Lee et al.,1994 25} & 22 & $\mathrm{~F}$ & 5 & $\mathrm{~S}$ & hematoma no núcleo lentiforme $\mathrm{E}$ \\
\hline & 23 & $\mathrm{~F}$ & 7 & $\mathrm{~S}$ & $\begin{array}{l}\text { fratura de crânio, } \\
\text { hematoma extradural E }\end{array}$ \\
\hline & 24 & $\mathrm{~F}$ & 7 & $\mathrm{~S}$ & hematoma extradural parietal $\mathrm{E}$ \\
\hline & 25 & $\mathrm{M}$ & 12 & M & antes da CT \\
\hline & 26 & M & 15 & $\mathrm{~S}$ & $\begin{array}{l}\text { fratura de crânio, hematoma no } \\
\text { núcleo lentiforme D }\end{array}$ \\
\hline & 27 & M & 17 & $\mathrm{~S}$ & hematoma talâmico D \\
\hline & 28 & M & 18 & $\mathrm{~S}$ & antes da CT \\
\hline & 29 & M & 20 & $\mathrm{~S}$ & contusão cerebral \\
\hline & 30 & $\mathrm{~F}$ & 20 & $\mathrm{~S}$ & $\begin{array}{l}\text { hemorragia occipital, } \\
\text { contusão temporal E }\end{array}$ \\
\hline & 31 & $\mathrm{~F}$ & 44 & $\mathrm{~S}$ & hematoma subdural bilateral \\
\hline
\end{tabular}

M, moderado; S, severo; ?, desconhecido; PE, putâmen esquerdo; NC, núcleo caudado; NL, núcleo lentiforme; BPCI, braço posterior da cápsula interna; GP, globo pálido; $\mathrm{CNCE}$, cabeça do núcleo caudado esquerdo; BACI, braço anterior da cápsula interna. 
Tabela 3. Trauma crânio-encefálico e distonia nos casos da Tabela 2.

\begin{tabular}{|c|c|c|c|}
\hline Caso & $\begin{array}{c}\text { Manifestações } \\
\text { neurológicas iniciais }\end{array}$ & $\begin{array}{l}\text { Intervalo entre } \\
\text { TCE e distonia }\end{array}$ & Distribuição da distonia \\
\hline 1 & hemiplegia $\mathrm{E}$ & $4 \mathrm{~s}$ & hemidistonia $\mathrm{E}$ \\
\hline 2 & ausente & $4 \mathrm{a}$ & distonia focal de MSE \\
\hline 3 & hemiparesia D, afasia & $6 a$ & hemidistonia D \\
\hline 4 & hemiparesia $\mathrm{E}$ & $6 \mathrm{~d}$ & hemidistonia $\mathrm{E}$ \\
\hline 5 & hemiparesia $\mathrm{E}$ & $16 \mathrm{~d}$ & hemidistonia $\mathrm{E}$ \\
\hline 6 & hemiparesia D & $1 \mathrm{~s}$ & hemidistonia D \\
\hline 7 & ausente & $1 \mathrm{~s}$ & hemidistonia D \\
\hline 8 & hemiparesia D, disartria & $1 \mathrm{~m}$ & hemidistonia D \\
\hline 9 & ausente & $24 \mathrm{~h}$ & distonia generalizada \\
\hline 10 & hemiparesia $\mathrm{E}$ & $14 \mathrm{~m}$ & hemidistonia $\mathrm{E}$ \\
\hline 11 & hemiparesia $\mathrm{E}$ & $4 \mathrm{a}$ & hemidistonia $\mathrm{E}$ \\
\hline 12 & ausente & $4 \mathrm{~d}$ & hemidistonia $\mathrm{E}$ \\
\hline 13 & ausente & $10 \mathrm{~s}$ & hemidistonia D \\
\hline 14 & $?$ & $5 \mathrm{a}$ & torcicolo \\
\hline 15 & $?$ & $1 \mathrm{a}$ & hemidistonia D \\
\hline 16 & $?$ & $4 \mathrm{a}$ & hemidistonia $\mathrm{E}$ \\
\hline 17 & $?$ & $3 \mathrm{~m}$ & hemidistonia D \\
\hline 18 & $?$ & $3 a$ & hemidistonia $\mathrm{E}$ \\
\hline 19 & $?$ & $6 \mathrm{~m}$ & hemidistonia D \\
\hline 20 & $?$ & $6 \mathrm{~m}$ & hemidistonia $\mathrm{D}$, torcicolo \\
\hline 21 & $?$ & $2 \mathrm{a}$ & hemidistonia D \\
\hline 22 & hiperreflexia D, paralisia central do VII & $1 \mathrm{a}$ & hemidistonia D \\
\hline 23 & hiperreflexia D & $9 \mathrm{a}$ & distonia de MSD \\
\hline 24 & hiperreflexia D & $2 \mathrm{a}$ & distonia generalizada \\
\hline 25 & hemiparesia D, Babinski D & $2 \mathrm{~s}$ & hemidistonia D \\
\hline 26 & paralisia central do VII E & $1 \mathrm{~m}$ & distonia de MSE \\
\hline 27 & hiperreflexia D e E & $5 \mathrm{~m}$ & distonia de MSD, MSE \\
\hline 28 & hemiparesia D & $6 a$ & hemidistonia D \\
\hline 29 & paralisia central VII D & $1 \mathrm{a}$ & hemidistonia D \\
\hline 30 & hemiparesia D, hiperreflexia D & $1 \mathrm{a}$ & hemidistonia D \\
\hline 31 & hemiplegia D & $3 \mathrm{a}$ & distonia multifocal \\
\hline
\end{tabular}

D, direita; E, esquerda; h, horas; d, dias; s, semanas; m, meses; a, anos. 
dias após atropelamento, em abril 1996, resultando em trauma crânio-encefálico (TCE) grave e fratura de fêmur direito, e permanência do paciente em coma durante quatro dias na UTI. Paciente é tabagista (20 cigarros/dia) e etilista (1/2 garrafa de aguardente/dia) há 23 anos e em uso de $2 \mathrm{mg} / \mathrm{dia}$ de clonazepam. Não houve intercorrências no período pré-natal. O parto via vaginal ocorrera em ambiente hospitalar, idade gestacional não conhecida, com peso aproximado de 2300 gramas, sem uso de forceps; porém cianótico, necessitando de incubadora e oxigênio por dois dias. Aleitamento materno até os três meses de idade, apresentando logo em seguida desidratação grave e desnutrição. Aos 6 anos sarampo e caxumba aos 18 anos, ambas sem complicações. O exame físico geral mostrava-se normal. No exame neurológico, observamos na atitude sentada movimentos involuntários de hiperextensão e torção da cabeça e pescoço para o lado direito; na atitude ortostática tinha movimentos involuntários de cabeça e pescoço, face voltada para a direita e lordose compensatória discreta; no leito os movimentos involuntários eram de menor amplitude e menor frequência, opistótono, e hipertrofia importante do músculo esternocleidomastoideo esquerdo. Marcha claudicante à esquerda, com movimentos de membros superiores de pequena amplitude, não alternados e assincrônicos aos dos membros inferiores, lordose e alargamento da base de sustentação, com movimentos involuntários de cabeça e pescoço. Os exames laboratoriais estavam dentro dos padrões de normalidade. A tomografia de crânio (CT) do dia 16-abril-1996 (pós trauma) mostrava higroma bilateral, pouco mais evidenciável à esquerda e edema cerebral difuso, mais importante à esquerda. A CT crânio de controle do dia 22-abril-1996 demonstrou absorção completa do higroma subdural e atrofia córtico-subcortical. No dia 19dezembro-1996, a CT crânio mostrava discreta assimetria ventrícular e pequena calcificação na região parietal do hemisfério cerebral esquerdo. Presença de retenção mucosa no seio maxilar esquerdo. Ressonância magnética de crânio (19-fevereiro-1997) mostrou atrofia discreta do parênquima cerebral.

\section{DISCUSSÃO}

Há mais de um século vem se confirmando estreita relação causa-efeito entre o TCE e as distonias, assim ocorre também com outras distúrbios de movimento. As distonias surgem, usualmente, logo após a lesão cerebral causada pelo TCE ou como parte integrante do quadro clínico inicial; nestes casos, observou-se que as distonias progridem em relação à severidade mais rapidamente do que o intervalo entre o TCE e o aparecimento da distonia. Por outro lado, as distonias podem ocorrer após período de latência significativamente variável. Acredita-se que o desenvolvimento tardio das distonias pós-traumáticas seja uma necessidade fisiopatológica dos mecanismos de reorganização do sistema nervoso central, sendo então formuladas hipóteses de que qualquer alteração nestes, como por exemplo aumento de radicais livres estimulado pela presença de ferro nas lesões vasculares putâmino-cápsulo-caudadas, interromperiam ou modificariam o processo bioquímicoanátomo-fisiológico dos circuitos dos gânglios da base.

Após revisão da literatura dos casos de distonia pós-traumática, elaboramos duas tabelas (Tabela 2 e 3) com o intuito de analisarmos as correlações entre sexo, idade na época do trauma, severidade do TCE, presença ou não de manifestações neurológicas iniciais e intervalo temporal entre o trauma e o aparecimento do distúrbio do movimento, assim como determinar qual o possível substrato anatômico responsável por ele.

Dos 31 casos revisados, 58,07\% (18 casos) eram do sexo masculino e 41,93\% (13 casos) do sexo feminino. Quanto à idade no momento do trauma, observamos que variou entre 2 e 62 anos (média 15,16 anos) com a seguinte distribuição: 18 infantes $(58,06 \%), 8$ adolescentes $(25,80 \%)$ e 5 adultos $(16,14 \%)$.

Dentre os 11 casos $(35,48 \%)$ que sofreram TCE de grau moderado, $4(36,36 \%)$ não apresentaram manifestações neurológicas iniciais e desenvolveram após intervalo temporal médio de 14 meses e 24 dias, hemidistonia ( 3 casos) e distonia focal (1 caso). Nos sete casos restantes $(63,64 \%)$, hemiplegia/paresia contralateral à lesão foi a manifestação neurológica inicial, seguindose de hemidistonia desses mesmos membros após intervalo médio de 8 meses e 10 dias.

Nos 20 casos em que o TCE foi severo, apenas um (5\%) não apresentou manifestação neurológica inicial e desenvolveu em 24 horas distonia generalizada, enquanto os demais 19 pacientes tiveram manifestações iniciais diversas tais como: hemiplegia/paresia, hiperreflexia, paralisia facial; apresentaram hemidistonia (13 casos), distonia generalizada ( 2 casos), distonia multifocal (2 casos) 
e distonia focal ( 3 casos) em intervalos médios de 2 anos e 80 dias, 12 meses, 1 ano e 7 meses e 4 anos e 6 meses, respectivamente.

Em nossa revisão observamos que todas as faixas etárias foram acometidas de maneira irregular e desigual no que concerne à distribuição e progressão da distonia, dependendo da severidade do trauma, manifestações neurológicas iniciais presentes e localização anatômica da lesão (Tabela 2).

É real a dificuldade em correlacionar o grau de TCE necessário para a indução dos movimentos involuntários; por outro lado, poderíamos afirmar que a duração do coma e a presença de manifestações neurológicas iniciais seriam fatores indicadores de que os movimentos involuntários apareceriam mais tardiamente. É importante salientar que deterioração das funções cognitivas não foi evidenciada em momento algum, o que exerce este papel diferencial importante com distúrbios neurológicos degenerativos ou metabólicos.

Nos movimentos distônicos que ocorrem imediatamente após o evento desencadeante ou após a melhora da manifestação neurológica inicial, observou-se a progressão em severidade mais rapidamente. O desenvolvimento e a persistência destes movimentos, anos após a lesão cerebral original, sugerem que eles ocorram por mudanças anatômica e/ou fisiológica que toma o lugar da lesão, e que a perda do potencial regenerativo se espalhe para os neurônios sãos ${ }^{8}$.

É inquestionável que a correspondência somatotópica entre os déficits neurológicos iniciais e os subsequentes movimentos involuntários tem uma relação causal muito estreita, porém não se pode afirmar tal certeza quanto à base fisiopatológica de tal relação. Em nosso levantamento notamos alta frequência de lesões talâmicas ou em gânglios da base, demonstrando a importância do circuito putâmino-pálido-talâmico na produção de distonias. Estudos neurofisiológicos ${ }^{9,10}$ ressaltam esta afirmação, demonstrando que a atividade muscular voluntária prolongada, excessiva e variável em pacientes com distonia sugere que os gânglios da base agem seletivamente apenas naqueles músculos apropriados para determinadas atividades e inibem aqueles que não são requeridos.

Os hematomas extra- e subdurais causando distonia tiveram importância significativa em nossa revisão. Poderíamos então associá-los à herniação transtentorial que produziria áreas focais de isquemia por compressão vascular ${ }^{11}$. A artéria coróidea anterior é comprimida contra o tentório, produzindo isquemia do putâmen contralateral e do globo pálido lateral. Durante a herniação também é comprimido o suprimento vascular do tálamo ${ }^{12}$.

Em recente estudo, Krauss e colaboradores ${ }^{13}$ demonstraram que, apesar dos distúrbios de movimento, persistentes ou transitórios, serem sequelas comuns do TCE, a distonia representaria uma pequena parcela deste grupo. Por fim, ressaltamos o torcicolo espasmódico como consequência raríssima do trauma e também algumas entre muitas dúvidas que pairam sob seus mecanismos fisiopatológicos: Qual a severidade do TCE necessária para produzir a distonia? Seriam as manifestações neurológicas iniciais e a duração do coma fatores indicativos de início mais tardio da distonia? O aparecimento de sinais de lesão de nervo craniano funcionaria como fator prognóstico? Qual seria o substrato anatômico/fisiológico da distonia nos casos em que os estudos de imagem são normais?

Reafirmamos portanto a necessidade de maiores estudos epidemiológicos dos distúrbios do movimento pós-traumáticos, principalmente em nosso meio, para buscar maiores esclarecimentos na tentativa de minimizar esta condição rara, porém desabilitante.

\section{REFERÊNCIAS}

1. Clarke E, Dewhurst K. An illustrated history of brain function. Los Angeles: Univ California Press, 1972:124-40.

2. Fahn S. Concept and classification of dystonia. In: Fahn S, Marsden CD, Calne DB (eds). Dystonia 2. Advances in Neurology. New York:Raven Press, 1988:50:1-8 .

3. Oppenheimmer H. Uber eine eigenartige Krampfrankheit des kindlichen und jungendichen Alters. Neurol Zbl 1911;30:1090.

4. Jankovic T, Fahn S. Dystonic syndromes. In:Jankovic J, Tolosa E (eds). Parkinson's disease and movement disorders. Baltimore: Urban-Scharzenberg, 1988:283-314.

5. Fahn S, Eldridge R. Defintion of dystonia and classification of dystonic states. Adv Neurol 1976;14:1-5. 
6. Jankovic J, Fahn S. Dystonic disorders. In Jankovic J, Tolosa E (eds):Parkinson's disease and movement disorders, 2.Ed. Baltimore: Willians \& Wilkins, 1993: 337-374.

7. Calne DB, Lange AE. Secundary dystonia. In Fahns, Marsen CD, Calned DB (eds). Dystonia 2. Advances in Neurology. New York; Raven Press 1988;50:9-33.

8. Raisman G. What hope for repair of the brain? Ann Neurol 1978, 3:101-106.

9. Hallet M, Khoshbin S. A physiological mechanism of bradykinesia. Brain 1980;103:301-314.

10. Van der Kamp A, Berardelli A, Thompson PD, Day BL, Marsden CD. Rapid elbow movements in patients with torsion dystonia. J Neurol Neurosurg Psychiatry 1989;52:1043-1049.

11. Lindenberg R. Compression pathogenetic factor for tissue necrosis and their areas of predilection. J Neuropathol Exp Neurol 1955;14:223-243.

12. Blackwwod WM, Minewin WR, Meyer A, Norman RM, Reissel DS. Greenfields neuropathology. 2.Ed. Baltimore:Willians \& Wilkins, 1991:540-576.

13. Krauss JK, Tränkle R, Kopp KH. Post-traumatic movement disorders in survivors of severe head injury. Neurology 1996;47:1488-1491.

14. Messimy R, Diebler C, Metzger J. Dystonie de torsion du membre superieur gauche probablement à un traumatisme cranien. Rev Neurol (Paris) 1977;133:199-206.

15. Burk RE, Fahn S, Gold AP. Delayed-onset dystonia in patients with "static" encephalopathy. J Neurol Neurosurg Psychiatry 1980;43:789-797.

16. Maki Y, Akimoto H, Enomoto T. Injuries of basal ganglia following trauma in children. Child's Brain 1980;7:113-123.

17. Mauro AJ, Fahn S, Russman B. Hemidystonia following "minor" head trauma [Abst]. Ann Neurol 1980;8:108.

18. Brett EM, Hoare RD. Progressive hemidystonia due to focal basal ganglia lesion after mild head trauma [Abstr]. J Neurol Neurosurg Psychiatry 1981;44:460.

19. Demierre B, Rondot P. Dystonia caused by putamino-capsulo-caudate vascular lesions. J Neurol Neurosurg Psychiatry 1983;46:404-409.

20. Elridge R. The torsion dystonias:literature review and genetic and clinical studies. Neurology 1970;20:1-78.

21. Pettigrew LC, Jankovic J. Hemidystonia:a report of 22 patients and a review of the literature. J Neurol Neurosurg Psychiatry 1985;48:650-657.

22. Eaton JM. Hemidystonia due to subdural hematoma. Neurology 1988;38:507.

23. Isaac K, Cohen JA. Post-trauamtic torticollis. Neurology 1989;39:1642-1643.

24. Krauss JK, Mohadjer M, Braus DF, Wakhloo AK, Nobbe F, Mundiger F. Dystonia following head trauma:a report of nine patients and a review of the literature. Mov Disord 1992;7:263-272.

25. Lee MS, Rinne JO, Ceballos-Baumann A, Thompson P, Marsden CD. Dystonia after head trauma. Neurology 1994;44:13741378 . 